\title{
Linear Alfvén waves in the solar atmosphere
}

\author{
K. Murawski ${ }^{1}$ and Z. E. Musielak ${ }^{2,3}$ \\ 1 Group of Astrophysics, Institute of Physics, UMCS, ul. Radziszewskiego 10, 20-031 Lublin, Poland \\ e-mail: kmur@kft.umcs.lublin.pl \\ 2 Department of Physics, University of Texas at Arlington, Arlington, TX 76019, USA \\ e-mail: zmusielak@uta.edu \\ 3 Kiepenheuer-Institut für Sonnenphysik, Schöneckstr. 6, Freiburg 79104, Germany \\ e-mail: musielak@kis.uni-freiburg.de
}

Received 10 March 2010 / Accepted 11 May 2010

\begin{abstract}
Aims. We aim to analytically and numerically explore small-amplitude Alfvén waves in the solar atmosphere. Methods. We transform the wave equations to obtain the cutoff-frequency and wave travel time for strictly linear Alfvén waves. The wave equations are solved numerically to find out spatial and temporal signatures of the waves.

Results. The analytical predictions are verified by numerically solving the wave equations for linear Alfvén waves. The waves are impulsively generated and their characteristics and behavior in the solar atmosphere are investigated by the numerical simulations. The derived cutoff-frequency is used to determine regions in the solar atmosphere where strong reflection occurs for Alfvén waves of different frequencies.

Conclusions. The numerical results reveal that impulsively generated small-amplitude waves exhibit characteristic spatial and temporal signatures which agree with the predictions of the analytical theory.
\end{abstract}

Key words. magnetohydrodynamics (MHD) - Sun: atmosphere

\section{Introduction}

The Sun is a compressible medium and so is able to support acoustic waves. However, the presence of a magnetic field introduces additional elasticity to the natural compressibility of the plasma. A magnetic field imparts magnetic pressure and tension forces, which sustain additional wave motion. Among others Alfvén waves are of great interest as they may participate in the heating of the solar corona (e.g., Hollweg 1991) and the acceleration of the solar wind (e.g., Parker 1991). As a result, the evolution of Alfvén waves in the solar atmosphere is a fundamental problem which requires further exploration. This problem is formidable though, because the solar atmosphere is a strongly inhomogeneous and structured medium (e.g., Zaqarashvili \& Murawski 2007).

We consider an atmospheric model for which the basic conditions for the propagation of linear Alfvén waves can be determined by an analytic theory. To verify the prediction of this theory, we numerically solve the resulting wave equations and compare the results of numerical simulations with those obtained analytically.

Some Alfvén waves originate from the dense photosphere where they can be reflected back into the photosphere, but some of them get through the cutoff-region and reach the solar corona (Musielak \& Moore 1995). Through the coupling provided by the magnetic field the solar chromosphere and corona host a large amount of these waves. Indeed, observational evidence of Alfvénic-like fluctuations in the corona and chromosphere (DePontieu et al. 2007; Tomczyk et al. 2007; Bonet et al. 2008; Fujimura \& Tsuneta 2009; Jess et al. 2009;
Wedemeyer-Böhm \& van der Voort 2009) indicate frequencies on the order of 3-9 min. However, some of these waves were interpreted by Van Doorsselaere et al. (2008a,b) as fast magnetoacoustic kink waves.

While there are several studies of the Alfvén wave propagation and reflection in stratified atmospheres that relies on the Fourier decomposition of an incident signal (e.g. Velli 1993), very few of them considered the problem for impulsively generated waves. For instance, Hollweg \& Isenberg (2007) showed that for impulsively generated Alfvén wave the dynamics could be understood in terms of the first two reflected signals. The cutoff-frequency criterion presented here offers an alternative approach to the usual Fourier analysis.

It has been shown that Alfvén waves may become nonlinear already in the chromosphere, exerting a ponderomotive force on magnetoacoustic waves (Suzuki \& Inutsuka 2005; Verdini \& Velli 2007; Verdini et al. 2009; Matsumoto \& Shibata 2010). Numerical simulations of Alfvén waves launched from the chromosphere reveal that high-frequency modes are attenuated before they reach the corona by coupling with magnetoacoustic waves (Matsumoto \& Shibata 2010). For our purpose here we only discuss small-amplitude Alfvén waves. Large-amplitude waves are left for future studies.

This paper is organized as follows. A physical model of the solar atmosphere is presented in Sect. 2. The following part of the paper discusses linear wave equations for Alfvén waves. Small-amplitude Alfvén waves are studied by numerical means in Sect. 4. The analytical and numerical results are discussed in Sect. 5. We conclude with a summary of the main results. 


\section{A physical model}

\subsection{MHD equations}

Our model system is taken to be composed of a gravitationallystratified solar atmosphere that is described by the ideal magnetohydrodynamic (MHD) equations

$$
\begin{aligned}
\frac{\partial \varrho}{\partial t}+\nabla \cdot(\varrho \boldsymbol{V}) & =0 \\
\varrho \frac{\partial \boldsymbol{V}}{\partial t}+\varrho(\boldsymbol{V} \cdot \nabla) \boldsymbol{V} & =-\nabla p+\frac{1}{\mu}(\nabla \times \boldsymbol{B}) \times \boldsymbol{B}+\varrho \boldsymbol{g} \\
\frac{\partial \boldsymbol{B}}{\partial t} & =\nabla \times(\boldsymbol{V} \times \boldsymbol{B}) \\
\nabla \cdot \boldsymbol{B} & =0 \\
\frac{\partial p}{\partial t}+\boldsymbol{V} \cdot \nabla p & =-\gamma p \nabla \cdot \boldsymbol{V} \\
p & =\frac{k_{\mathrm{B}}}{m} \varrho T .
\end{aligned}
$$

Here $\varrho$ is the mass density, $\boldsymbol{V}$ is the flow velocity, $\boldsymbol{B}$ is the magnetic field, $p$ is the gas pressure, $\gamma=5 / 3$ is the adiabatic index, $\boldsymbol{g}=(0,-g, 0)$ is a vector of gravitational acceleration with its value $g=274 \mathrm{~m} \mathrm{~s}^{-2}, T$ is the temperature, $m$ is the mean particle mass and $k_{\mathrm{B}}$ is Boltzmann's constant.

\subsection{A model of the solar atmosphere}

We assume that the solar atmosphere is in static equilibrium $\left(\boldsymbol{V}_{\mathrm{e}}=0\right)$ with a force-free magnetic field, i.e.

$\left(\nabla \times \boldsymbol{B}_{\mathrm{e}}\right) \times \boldsymbol{B}_{\mathrm{e}}=0$.

Henceforth the subscript $\mathrm{e}_{\mathrm{e}}$ corresponds to equilibrium quantities. As a result, the pressure gradient is balanced by the gravity force

$-\nabla p_{\mathrm{e}}+\varrho_{\mathrm{e}} \boldsymbol{g}=0$.

Using the ideal gas law of Eq. (6) and the $y$-component of the hydrostatic pressure balance indicated by Eq. (8), we express the equilibrium gas pressure and mass density as

$p_{\mathrm{e}}(y)=p_{0} \exp \left(-\int_{y_{\mathrm{r}}}^{y} \frac{\mathrm{d} y^{\prime}}{\Lambda_{\mathrm{p}}\left(y^{\prime}\right)}\right), \quad \varrho_{\mathrm{e}}(y)=\frac{p_{\mathrm{e}}(y)}{g \Lambda_{\mathrm{p}}(y)}$.

Here

$\Lambda_{\mathrm{p}}(y)=\frac{k_{\mathrm{B}} T_{\mathrm{e}}(y)}{m g}$

is the pressure scale-height, and $p_{0}$ denotes the gas pressure at the reference level that is set and hold fixed at $y_{\mathrm{r}}=10 \mathrm{Mm}$.

We adopt a smoothed step-function profile for the plasma temperature

$T_{\mathrm{e}}(y)=\frac{1}{2} T_{\mathrm{c}}\left[1+d_{\mathrm{t}}+\left(1-d_{\mathrm{t}}\right) \tanh \left(\frac{y-y_{\mathrm{t}}}{y_{\mathrm{w}}}\right)\right]$,

where $d_{\mathrm{t}}=T_{\mathrm{ch}} / T_{\mathrm{c}}$ and $T_{\mathrm{ch}}$ denote the chromospheric temperature at its lower part. The symbol $T_{\mathrm{c}}$ corresponds to the temperature of the solar corona that is separated from the chromosphere by the transition region that is located at $y_{\mathrm{t}}=2 \mathrm{Mm}$. A width of the transition region is $y_{\mathrm{w}}=200 \mathrm{~km}$. We set and hold fixed $T_{\mathrm{c}}=200 T_{\mathrm{ch}}$.

According to Eq. (9) the temperature profile of Eq. (11) uniquely determines equilibrium mass density and gas pressure
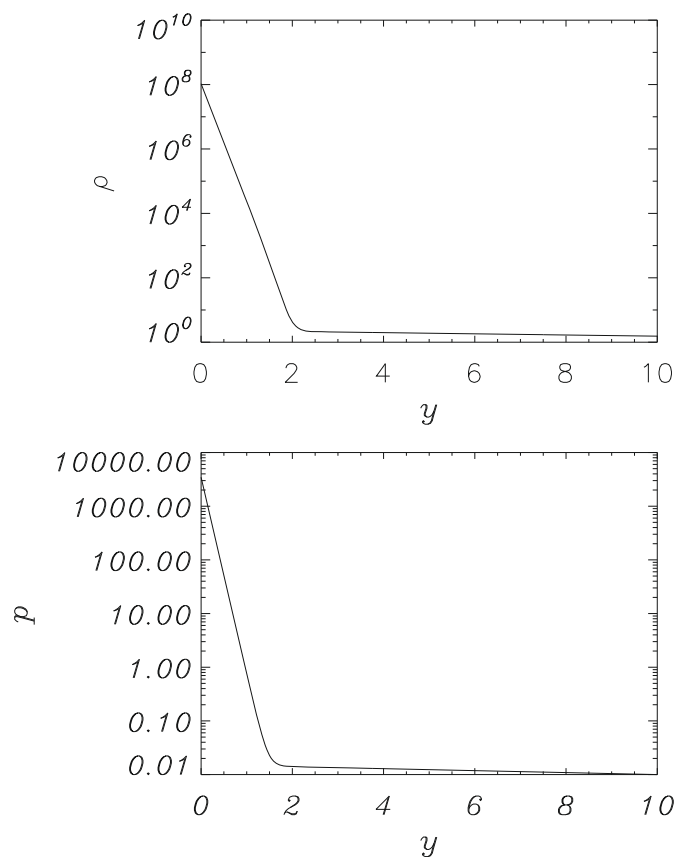

Fig. 1. Equilibrium mass density (top panel) and gas pressure (bottom panel) in units of $10^{-12} \mathrm{~kg} \mathrm{~m}^{-3}$ and $1 \mathrm{~Pa}$, respectively. The distance $y$ is expressed in $1 \mathrm{Mm}$.

profiles (Fig. 1). Both $\varrho_{\mathrm{e}}(y)$ and $p_{\mathrm{e}}(y)$ experience a sudden falloff below the transition region.

We consider a uniform vertical magnetic field such as

$\boldsymbol{B}_{\mathrm{e}}=B_{\mathrm{e}} \hat{\boldsymbol{y}}, \quad B_{\mathrm{e}}=$ const.

Here $\hat{\boldsymbol{y}}$ is a unit vector along the $y$-direction. This choice of $\boldsymbol{B}_{\mathrm{e}}$ results in Eq. (7) being satisfied and the Alfvén speed, $c_{\mathrm{A}}$, is given as

$c_{\mathrm{A}}(y)=\frac{B_{e}}{\sqrt{\mu \varrho_{\mathrm{e}}(y)}}$

whose profile is displayed in Fig. 2 (top panel). Note that in the low chromosphere $c_{\mathrm{A}}(y=1 \mathrm{Mm}) \simeq 10 \mathrm{~km} \mathrm{~s}^{-1}$. It raises up abruptly through the transition region, reaching a value of $c_{\mathrm{A}}(y=10 \mathrm{Mm}) \simeq 1050 \mathrm{~km} \mathrm{~s}^{-1}$ in the solar corona. The growth of $c_{\mathrm{A}}(y)$ results from the fall-off of $\varrho_{\mathrm{e}}$ with height. For comparison purposes the sound speed,

$c_{\mathrm{s}}(y)=\sqrt{\frac{\gamma p_{\mathrm{e}}}{\varrho_{\mathrm{e}}}}$,

is shown in Fig. 2 (bottom panel). The sound speed reaches a value of about $7 \mathrm{~km} \mathrm{~s}^{-1}$ below the altitude $y=1 \mathrm{Mm}$ and it grows to about $105 \mathrm{~km} \mathrm{~s}^{-1}$ in the solar corona, for $y>3 \mathrm{Mm}$.

As a result of the Alfvén and sound speed profiles the plasma

$\beta=\frac{2 \mu p_{\mathrm{e}}}{B_{\mathrm{e}}^{2}}=\frac{2}{\gamma} \frac{c_{\mathrm{s}}^{2}}{c_{\mathrm{A}}^{2}}$

is illustrated in Fig. 3. Note that $\beta \simeq 0.5$ for $y=1 \mathrm{Mm}$ and it falls off abruptly at high altitudes reaching a value of $\beta \simeq 1.2 \times 10^{-2}$ at $y=10 \mathrm{Mm}$. 
K. Murawski and Z. E. Musielak: Alfvén waves in the solar atmosphere
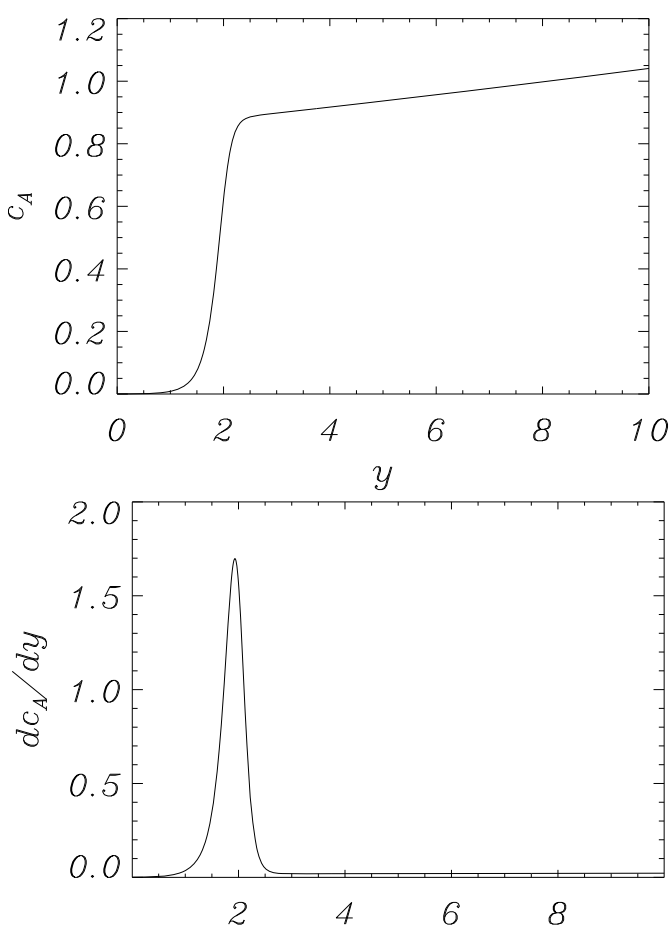

y

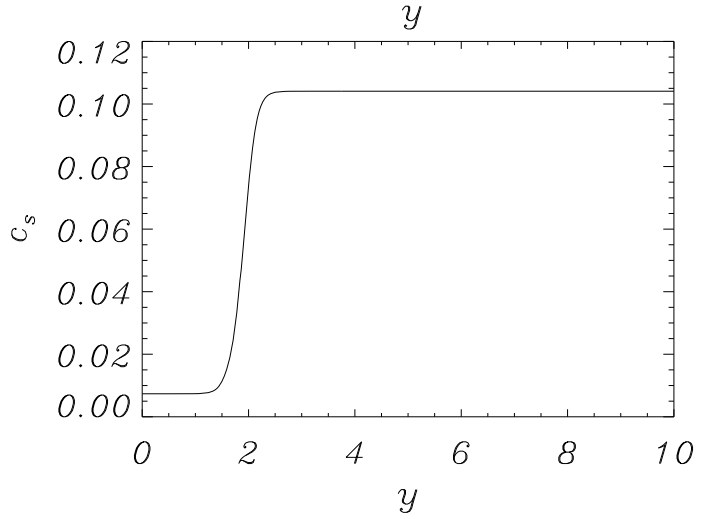

Fig. 2. Equilibrium profile of Alfvén speed $c_{\mathrm{A}}$ (top panel), $\mathrm{d} c_{\mathrm{A}} / \mathrm{d} y$ (middle panel) and sound speed (bottom panel). These speeds, $\mathrm{d} c_{\mathrm{A}} / \mathrm{d} y$ and the distance $y$ are expressed in units of $1 \mathrm{Mm} \mathrm{s}^{-1}, 1 \mathrm{~s}^{-1}$ and $1 \mathrm{Mm}$, respectively.

\section{Linear Alfvén waves}

\subsection{Wave equations for Alfvén waves}

We now discuss small-amplitude perturbations of the equilibrium that is described in Sect. 2.2. Assuming that plasma evolution occurs only along the $y$-direction, the $z$-components of momentum Eq. (2) and induction Eq. (3) can be linearized, leading to the following equations:

$$
\begin{aligned}
\varrho_{\mathrm{e}}(y) \frac{\partial V_{\mathrm{z}}}{\partial t} & =B_{\mathrm{e}} \frac{\partial B_{\mathrm{z}}}{\partial y}, \\
\frac{\partial B_{\mathrm{z}}}{\partial t} & =B_{\mathrm{e}} \frac{\partial V_{\mathrm{z}}}{\partial y} .
\end{aligned}
$$

Here $V_{\mathrm{z}}$ and $B_{\mathrm{z}}$ denote the perturbed $z$-components of the plasma velocity and magnetic field, respectively. Hence we arrive at the following wave equations:

$$
\frac{\partial^{2} V_{\mathrm{z}}}{\partial t^{2}}=c_{\mathrm{A}}^{2}(y) \frac{\partial^{2} V_{\mathrm{z}}}{\partial y^{2}},
$$

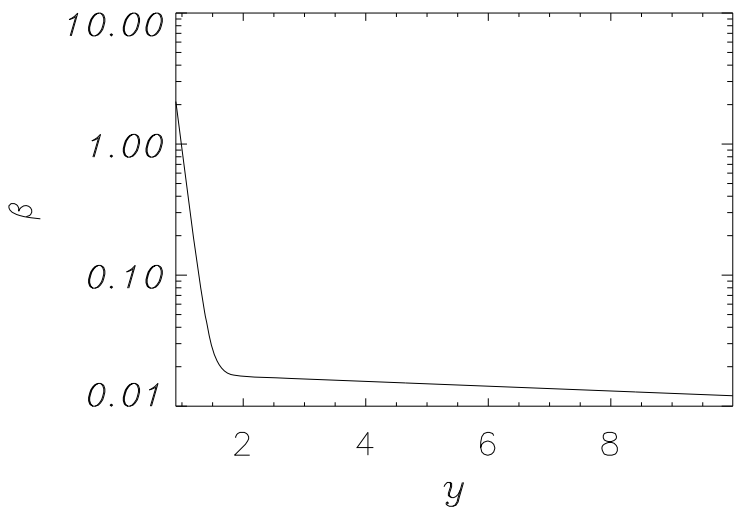

Fig. 3. Equilibrium profile of the plasma $\beta$ vs. height $y$ (in $1 \mathrm{Mm}$ ).

$\frac{\partial^{2} B_{\mathrm{z}}}{\partial t^{2}}=\frac{\partial}{\partial y}\left(c_{\mathrm{A}}^{2}(y) \frac{\partial B_{\mathrm{z}}}{\partial y}\right)$

Note that $V_{z}$ and $B_{z}$ are described by different wave equations, which means that there is a phase shift between the wave variables and, as a result, they behave differently. The derived wave equations have non-constant coefficients and become the classical wave equations with constant coefficients when a homogeneous plasma with $c_{\mathrm{A}}^{2}(y)=$ const is considered.

\subsection{Cutoff-frequency with approximate wave travel time}

We rewrite the wave equations given by Eqs. (18) and (19) in their standard form, i.e., without the first derivative term; the wave equations written in their standard forms are often referred to as Klein-Gordon equations (e.g., Rae \& Roberts 1982; Musielak et al. 1987, 2006). Because there is no term with the first derivative in the wave equation for $V_{z}$, this equation is already written in its standard form. However, in order to cast the wave equation for $B_{z}$ in its standard form, we must use the transformation

$B_{z}(y, t)=B_{1 z}(y, t) \exp \left[-\int^{y} \frac{1}{c_{\mathrm{A}}}\left(\frac{\mathrm{d} c_{\mathrm{A}}}{\mathrm{d} \tilde{y}}\right) \mathrm{d} \tilde{y}\right]$.

The resulting standard wave equation can be written as

$\frac{\partial^{2} B_{1 z}}{\partial t^{2}}-c_{\mathrm{A}}^{2}(y) \frac{\partial^{2} B_{1 z}}{\partial y^{2}}+\Omega_{\mathrm{crit}}^{2}(y) B_{1 z}=0$,

where $\Omega_{\text {crit }}$ is the so-called critical frequency (e.g., Musielak et al. 1992, 2006) given by

$\Omega_{\text {crit }}^{2}(y)=c_{\mathrm{A}}\left(\frac{\mathrm{d}^{2} c_{\mathrm{A}}}{\mathrm{d} y^{2}}\right)$.

Note that the critical frequency for $V_{z}$ is zero.

We make Fourier transforms in time $V_{z}(y, t)=\tilde{V}_{z}(y) \mathrm{e}^{-i \omega t}$ and $B_{1 z}(y, t)=\tilde{B}_{1 z}(y) \mathrm{e}^{-i \omega t}$, and obtain

$\frac{\mathrm{d}^{2} \tilde{V}_{z}}{\mathrm{~d} y^{2}}+\frac{\omega^{2}}{c_{\mathrm{A}}^{2}(y)} \tilde{V}_{z}=0$,

and

$\frac{\mathrm{d}^{2} \tilde{B}_{1 z}}{\mathrm{~d} y^{2}}+\frac{\omega^{2}-\Omega_{\text {crit }}^{2}(y)}{c_{\mathrm{A}}^{2}(y)} \tilde{B}_{1 z}=0$.

The transformed wave equations are in the form of Euler's equation $u^{\prime \prime}+\left(C_{\mathrm{E}} / 4 x^{2}\right) u=0$, where $u^{\prime \prime}$ is the second derivative of 
$u$ with respect to $x$. This equation has the oscillatory and nonoscillatory solutions when $C_{\mathrm{E}}>1$ and $C_{\mathrm{E}}<1$, respectively, and it has the turning-point that separates these solutions when $C_{\mathrm{E}}=1$ (e.g., Murphy 1960).

We define the turning-point frequencies $\Omega_{\mathrm{tp}, \mathrm{V}}$ and $\Omega_{\mathrm{tp}, \mathrm{B}}$ for $\tilde{V}_{z}$ and $\tilde{B}_{1 z}$, respectively, by taking the non-constant coefficients in Eqs. (23) and (24) to be equal to the term 1/4 $y^{2}$ from Euler's equation (see Musielak et al. 2006). The results are

$\Omega_{\mathrm{tp}, \mathrm{V}}^{2}(y)=\frac{c_{\mathrm{A}}^{2}(y)}{4 y^{2}}$,

and

$\Omega_{\mathrm{tp}, \mathrm{B}}^{2}(y)=\Omega_{\mathrm{crit}}^{2}(y)+\frac{c_{\mathrm{A}}^{2}(y)}{4 y^{2}}$.

Note that the obtained turning-point frequencies depend on the approximate wave travel time (Routh et al. 2010), which is defined as

$t_{\mathrm{ap}}=\frac{\left|y-y_{0}\right|}{c_{\mathrm{A}}(y)}$.

Here $y_{0}$ denotes an atmospheric height at which waves are initially (at $t=0 \mathrm{~s}$ ) launched. To distinguish between $t_{\text {ap }}$ and the actual wave travel time, the latter will be introduced in the next subsection.

Using the oscillation theorem (e.g., Kahn 1990), it is easy to show that the conditions $\omega>\Omega_{\mathrm{tp}, \mathrm{V}}$ and $\omega>\Omega_{\mathrm{tp}, \mathrm{B}}$ give oscillatory (or propagating wave) solutions for the corresponding wave variables. Obviously, the solutions are non-oscillatory (or non-propagating waves) when $\omega<\Omega_{\mathrm{tp}, \mathrm{V}}$ and $\omega<\Omega_{\mathrm{tp}, \mathrm{B}}$.

After obtaining the turning-point frequencies for both wave variables, we now follow Musielak et al. (2006) and select the cutoff-frequency $\Omega_{\text {cut,y }}$ from the condition

$\Omega_{\text {cut }, \mathrm{y}}(y)=\left[\max \left(\Omega_{\mathrm{tp}, \mathrm{V}}^{2}(y), \Omega_{\mathrm{tp}, \mathrm{B}}^{2}(y)\right)\right]^{1 / 2}$.

\subsection{Cutoff-frequency with actual wave travel time}

To obtain a cutoff-frequency that involves the actual wave travel time $t_{\mathrm{ac}}$, we follow Musielak et al. (1992) and introduce

$\mathrm{d} \tau=\frac{\mathrm{d} y}{c_{\mathrm{A}}(y)}$,

which allows us to write Eqs. (18) and (19) in the following forms:

$\frac{\partial^{2} V_{z}}{\partial t^{2}}-\frac{\partial^{2} V_{z}}{\partial \tau^{2}}+\omega_{\mathrm{A}} \frac{\partial V_{z}}{\partial \tau}=0$

$\frac{\partial^{2} B_{z}}{\partial t^{2}}-\frac{\partial^{2} B_{z}}{\partial \tau^{2}}-\omega_{\mathrm{A}} \frac{\partial B_{z}}{\partial \tau}=0$

Here

$\omega_{\mathrm{A}}(\tau)=\frac{1}{c_{\mathrm{A}}} \frac{\mathrm{d} c_{\mathrm{A}}}{\mathrm{d} \tau}$

Using the transformations

$V_{z}(t, \tau)=v(t, \tau) \exp \left[\frac{1}{2} \int^{\tau} \omega_{\mathrm{A}}(\tilde{\tau}) d \tilde{\tau}\right]$,

and

$B_{z}(t, \tau)=b(t, \tau) \exp \left[-\frac{1}{2} \int^{\tau} \omega_{\mathrm{A}}(\tilde{\tau}) \mathrm{d} \tilde{\tau}\right]$, we cast the wave equations given by Eqs. (18) and (19) in their standard forms

$\frac{\partial^{2} v}{\partial t^{2}}-\frac{\partial^{2} v}{\partial \tau^{2}}+\Omega_{\mathrm{cr}, \mathrm{v}}^{2}(\tau) v=0$

and

$\frac{\partial^{2} b}{\partial t^{2}}-\frac{\partial^{2} b}{\partial \tau^{2}}+\Omega_{\mathrm{cr}, \mathrm{b}}^{2}(\tau) b=0$

where the critical frequencies are defined as

$\Omega_{\mathrm{cr}, \mathrm{v}}^{2}(\tau)=\frac{1}{2}\left[\frac{1}{2} \omega_{\mathrm{A}}^{2}-\frac{\mathrm{d} \omega_{\mathrm{A}}}{\mathrm{d} \tau}\right]$,

and

$\Omega_{\mathrm{cr}, \mathrm{b}}^{2}(\tau)=\frac{1}{2}\left[\frac{1}{2} \omega_{\mathrm{A}}^{2}+\frac{\mathrm{d} \omega_{\mathrm{A}}}{\mathrm{d} \tau}\right]$

The critical frequencies can also be expressed in terms of the Alfvén speed, $c_{\mathrm{A}}$, and its derivatives. Using Eq. (32), we obtain

$\Omega_{\mathrm{cr}, \mathrm{v}}^{2}(\tau)=\frac{1}{2}\left[\frac{3}{2} \frac{1}{c_{\mathrm{A}}^{2}}\left(\frac{\mathrm{d} c_{\mathrm{A}}}{\mathrm{d} \tau}\right)^{2}-\frac{1}{c_{\mathrm{A}}}\left(\frac{\mathrm{d}^{2} c_{\mathrm{A}}}{\mathrm{d} \tau^{2}}\right)\right]$,

and

$\Omega_{\mathrm{cr}, \mathrm{b}}^{2}(\tau)=\frac{1}{2}\left[\frac{1}{c_{\mathrm{A}}}\left(\frac{\mathrm{d}^{2} c_{\mathrm{A}}}{\mathrm{d} \tau^{2}}\right)-\frac{1}{2} \frac{1}{c_{\mathrm{A}}^{2}}\left(\frac{\mathrm{d} c_{\mathrm{A}}}{\mathrm{d} \tau}\right)^{2}\right]$.

It is interesting to note that the above expressions for the critical frequencies are the same as those obtained by Routh et al. (2010), who studied the propagation of torsional waves along solar magnetic flux tubes. This clearly implies that the physical properties of torsional tube waves and the Alfvén waves investigated here are the same.

After obtaining the critical frequencies, we make Fourier transforms in time $v(\tau, t)=\tilde{v}(\tau) \mathrm{e}^{-i \omega t}$ and $b(\tau, t)=\tilde{b}(\tau) \mathrm{e}^{-i \omega t}$, and obtain

$\frac{\mathrm{d}^{2} \tilde{v}}{\mathrm{~d} \tau^{2}}+\left[\omega^{2}-\Omega_{\mathrm{cr}, \mathrm{v}}^{2}(\tau)\right] \tilde{v}=0$

and

$\frac{\mathrm{d}^{2} \tilde{b}}{\mathrm{~d} \tau^{2}}+\left[\omega^{2}-\Omega_{\mathrm{cr}, \mathrm{b}}^{2}(\tau)\right] \tilde{b}=0$

We use these equations and follow the approach described in the previous subsection to define the turning-point frequencies $\Omega_{\mathrm{tp}, \mathrm{v}}$ and $\Omega_{\mathrm{tp}, \mathrm{b}}$ for the transformed wave variables $\tilde{v}$ and $\tilde{b}$, respectively. The results are

$\Omega_{\mathrm{tp}, \mathrm{v}}^{2}(\tau)=\Omega_{\mathrm{cr}, \mathrm{v}}^{2}(\tau)+\frac{1}{4 \tau^{2}}$,

$\Omega_{\mathrm{tp}, \mathrm{b}}^{2}(\tau)=\Omega_{\mathrm{cr}, \mathrm{b}}^{2}(\tau)+\frac{1}{4 \tau^{2}}$.

It must be pointed out that both turning-point frequencies are expressed in terms of $\tau$, which is the actual wave travel time $t_{\mathrm{ac}}$ in an inhomogeneous medium

$\tau(y)=\int^{y} \frac{\mathrm{d} \tilde{y}}{c_{\mathrm{A}}(\tilde{y})}+\tau_{\mathrm{c}}=t_{\mathrm{ac}}(y)$

Here $\tau_{\mathrm{c}}$ is the integration constant to be determined from a given model of the background medium. 
Based on the oscillation theorem (e.g., Kahn 1990), the wave propagation conditions are $\omega>\Omega_{\mathrm{tp}, \mathrm{v}}$ and $\omega>\Omega_{\mathrm{tp}, \mathrm{b}}$. These conditions require that both wave variables are described by the propagating wave solutions and can be simplified by introducing the cutoff-frequency $\Omega_{\mathrm{cut}, \tau}$ given by

$\Omega_{\mathrm{cut}, \tau}(\tau)=\left[\max \left(\Omega_{\mathrm{tp}, \mathrm{v}}^{2}(\tau), \Omega_{\mathrm{tp}, \mathrm{b}}^{2}(\tau)\right)\right]^{1 / 2}$.

It is important to point out that the resulting cutoff-frequency $\Omega_{\text {cut }, \tau}$ depends on the actual wave travel time and that $\Omega_{\text {cut }, \tau}$ can only be determined once a model of the background medium is specified.

\subsection{The cutoff-frequency}

In the previous two subsections, we derived two different cutofffrequencies $\Omega_{\text {cut,y }}$ and $\Omega_{\text {cut }, \tau}$. We obtained the cutoff-frequency $\Omega_{\text {cut,y }}$ from the transformed wave equations that describe the wave propagation using the variable $y$, and we demonstrated that this cutoff explicitly depends on the approximate wave travel time. On the other hand, the cutoff-frequency $\Omega_{\text {cut }, \tau}$ was obtained from another set of transformed wave equations that involved the variable $\tau$, which represents the actual wave travel time. There were also significant differences between the critical frequencies for these two cases.

With two different cutoff-frequencies, we must now determine which one is the true cutoff-frequency that correctly describes the propagation conditions for the considered linear Alfvén waves. Because $\Omega_{\text {cut }, \tau}$ correctly accounts for the actual wave travel time, we choose it to be the true cutoff-frequency. Hence, we have $\Omega_{\text {cutoff }}=\Omega_{\text {cut }, \tau}$ or

$\Omega_{\text {cutoff }}(\tau)=\left[\max \left(\Omega_{\mathrm{tp}, \mathrm{v}}^{2}(\tau), \Omega_{\mathrm{tp}, \mathrm{b}}^{2}(\tau)\right)\right]^{1 / 2}$.

Once the true cutoff-frequency is determined, we may convert $\tau$ into $y$ and give the explicit form of $\Omega_{\text {cutoff }}(y)$. The result is

$\Omega_{\text {cutoff }}(y)=\left[\max \left(\Omega_{\text {tp }, \mathrm{v}}^{2}(y), \Omega_{\mathrm{tp}, \mathrm{b}}^{2}(y)\right)\right]^{1 / 2}$,

where the turning-point frequencies are given by

$\Omega_{\mathrm{tp}, \mathrm{v}}^{2}(y)=\Omega_{\mathrm{cr}, \mathrm{v}}^{2}(y)+\frac{1}{4 t_{\mathrm{ac}}^{2}(y)}$,

and

$\Omega_{\mathrm{tp}, \mathrm{b}}^{2}(y)=\Omega_{\mathrm{cr}, \mathrm{b}}^{2}(y)+\frac{1}{4 t_{\mathrm{ac}}^{2}(y)}$,

with the actual wave travel time $t_{\mathrm{ac}}$ defined by Eq. (45). In addition, the explicit forms of the critical frequencies are

$\Omega_{\mathrm{cr}, \mathrm{v}}^{2}(y)=\frac{1}{2}\left[\frac{1}{2}\left(\frac{\mathrm{d} c_{\mathrm{A}}}{\mathrm{d} y}\right)^{2}-c_{\mathrm{A}}\left(\frac{\mathrm{d}^{2} c_{\mathrm{A}}}{\mathrm{d} y^{2}}\right)\right]$,

$\Omega_{\mathrm{cr}, \mathrm{b}}^{2}(y)=\frac{1}{2}\left[\frac{1}{2}\left(\frac{\mathrm{d} c_{\mathrm{A}}}{\mathrm{d} y}\right)^{2}+c_{\mathrm{A}}\left(\frac{\mathrm{d}^{2} c_{\mathrm{A}}}{\mathrm{d} y^{2}}\right)\right]$.

Now, the cutoff-frequency can be obtained when we know which turning-point frequency is higher. This can only be determined when a model of background atmosphere is specified. This model is given in Sect. 2, and we will use it below to obtain the cutoff-frequency for linear Alfvén waves propagating along uniform magnetic field embedded in an non-isothermal atmosphere.

\subsection{Physical model considered in this paper}

We consider a stratified and non-isothermal medium with a uniform background magnetic field (for details, see Sect. 2.2). Let us introduce the density scale height $\Lambda_{d}$, which is defined as

$\frac{1}{\Lambda_{\mathrm{d}}}=-\frac{1}{\rho_{\mathrm{e}}} \frac{\mathrm{d} \rho_{\mathrm{e}}}{\mathrm{d} y}$

Note that for the background medium considered here, the density and pressure scale heights are different (see however Sect. 3.6).

Using the above definition, we obtain

$\frac{\mathrm{d} c_{\mathrm{A}}}{\mathrm{d} y}=\frac{c_{\mathrm{A}}}{2 \Lambda_{\mathrm{d}}}$

and

$\frac{\mathrm{d}^{2} c_{\mathrm{A}}}{\mathrm{d} y^{2}}=\frac{c_{\mathrm{A}}}{4 \Lambda_{\mathrm{d}}^{2}}\left[1-2\left(\frac{\mathrm{d} \Lambda_{\mathrm{d}}}{\mathrm{d} y}\right)\right]$.

Hence, the critical frequencies (see Eqs. (51) and (52)) can be written as

$\Omega_{\mathrm{cr}, \mathrm{v}}^{2}(y)=\Omega_{\mathrm{A}}^{2}(y)\left[\left(\frac{\mathrm{d} \Lambda_{\mathrm{d}}}{\mathrm{d} y}\right)-\frac{1}{4}\right]$

and

$\Omega_{\mathrm{cr}, \mathrm{b}}^{2}(y)=\Omega_{\mathrm{A}}^{2}(y)\left[\frac{3}{4}-\left(\frac{\mathrm{d} \Lambda_{\mathrm{d}}}{\mathrm{d} y}\right)\right]$,

where

$\Omega_{\mathrm{A}}^{2}(y)=\frac{c_{\mathrm{A}}^{2}(y)}{4 \Lambda_{\mathrm{d}}^{2}(y)}$.

The above equations clearly show that in the considered model either $\Omega_{\mathrm{cr}, \mathrm{v}}^{2}$ and $\Omega_{\mathrm{cr}, \mathrm{b}}^{2}$ are both positive, or at least one of them is positive and the other one is negative. To demonstrate this, we introduce $\Lambda_{\mathrm{d}}^{\prime}=d \Lambda_{\mathrm{d}} / \mathrm{d} y$ and consider two cases: $\Lambda_{\mathrm{d}}^{\prime}>0$ and $\Lambda_{\mathrm{d}}^{\prime}<0$. In the first case, we have $\Omega_{\mathrm{cr}, \mathrm{v}}^{2}>0$ and $\Omega_{\mathrm{cr}, \mathrm{b}}^{2}>0$ when $1<4 \Lambda_{\mathrm{d}}^{\prime}<3, \Omega_{\mathrm{cr}, \mathrm{v}}^{2}<0$ and $\Omega_{\mathrm{cr}, \mathrm{b}}^{2}>0$ when $4 \Lambda_{\mathrm{d}}^{\prime}<1$, and $\Omega_{\mathrm{cr}, \mathrm{v}}^{2}>0$ and $\Omega_{\mathrm{cr}, \mathrm{b}}^{2}<0$ when $4 \Lambda_{\mathrm{d}}^{\prime}>3$. In the second case, we always have $\Omega_{\mathrm{cr}, \mathrm{v}}^{2}<0$ and $\Omega_{\mathrm{cr}, \mathrm{b}}^{2}>0$. An interesting result is that no temperature distribution in this model can lead to both critical frequencies being negative.

For the temperature distribution given by Eq. (11), the density scale height can be written as

$\frac{1}{\Lambda_{\mathrm{d}}(y)}=\frac{1}{\Lambda_{\mathrm{p}}(y)}\left[1+\frac{H_{\mathrm{ct}}}{y_{\mathrm{w}}} \cosh ^{-2}\left(\frac{y-y_{\mathrm{t}}}{y_{\mathrm{w}}}\right)\right]$,

where the pressure scale height is given by Eq. (10) and $H_{\mathrm{ct}}=$ $c_{\mathrm{sc}}^{2} / \gamma g=$ const., with $c_{\mathrm{sc}}$ the sound speed that corresponds to the fixed temperature $T_{\mathrm{ct}}=T_{\mathrm{c}}\left(1-\mathrm{d}_{\mathrm{t}}\right) / 2$ (see Eq. (11)). In addition, the derivative of the density scale height with respect to $y$ is

$\frac{\mathrm{d} \Lambda_{\mathrm{d}}}{\mathrm{d} y}=\left(1-\frac{\Lambda_{\mathrm{d}}}{\Lambda_{\mathrm{p}}}\right)\left[1+2 \frac{\Lambda_{\mathrm{d}}}{y_{\mathrm{w}}} \tanh \left(\frac{y-y_{\mathrm{t}}}{y_{\mathrm{w}}}\right)\right]$.

Using Eqs. (59) and (60), we obtain the critical frequencies and then determine the cutoff-frequency with the help of Eq. (48). Hence we get the Alfvén cutoff-wave period,

$P_{\text {cutoff }}=\frac{2 \pi}{\Omega_{\text {cutoff }}}$ 


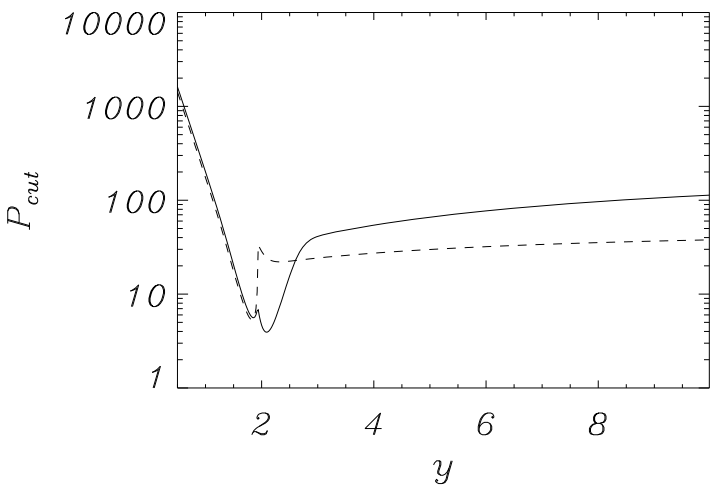

Fig. 4. Alfvén cutoff-wave period $P_{\text {cutoff }}$ of Eq. (61) (solid line) and its approximate analog of Eq. (28) (dashed line) in units of $1 \mathrm{~s}$. Distance $y$ is expressed in units of $1 \mathrm{Mm}$.

This cutoff-period is displayed in Fig. 4 (solid line) and its approximate analog $P_{\text {cut,y }}=2 \pi / \Omega_{\text {cut,y }}$ is illustrated by a dashed line. In the low chromosphere $P_{\text {cutoff }} \simeq 100 \mathrm{~s}$ at $y \simeq 1.1 \mathrm{Mm}$ and at higher levels it falls off abruptly with $y$ reaching a minimum $P_{\text {cutoff }} \simeq 4 \mathrm{~s}$ around the transition region. At higher altitudes it raises up, reaching a value of $P_{\text {cutoff }} \simeq 100 \mathrm{~s}$ at $y=8.5 \mathrm{Mm}$. Note that $P_{\text {cut,y }}$ lies close to $P_{\text {cutoff }}$ for $y<2 \mathrm{Mm}$, but it departs from $P_{\text {cutoff }}$ in the solar corona.

Note here that a different approach in literature is based on a use of the Elsässer variables (Velli 1993; Hollweg \& Isenberg 2007; Verdini \& Velli 2007; Verdini et al. 2009). For instance, Velli (1993) used a Fourier approach, demonstrating that wave reflection is a global phenomenon. It is interesting that a rough estimate of the cutoff-frequency, i.e. comparing the wave frequency with the maximum of the Alfvén speed gradient (e.g. Eq. (4) of Velli 1993), yields a period of about $7 \mathrm{~s}$, which is not so different from the value of about $4 \mathrm{~s}$ obtained from Fig. 4 at the transition region.

The Alfvén cutoff-period plotted in Fig. 4 can be used to determine the height in the solar atmosphere at which wave reflection becomes dominant. The condition for this height is $P_{\mathrm{w}}=P_{\text {cutoff }}$, where $P_{\mathrm{w}}$ is the wave period. For example, the reflection of Alfvén waves with a period of $80 \mathrm{~s}$ becomes important in the solar chromosphere at $y=1 \mathrm{Mm}$ and in the solar corona at $y=6 \mathrm{Mm}$. The situation is different for short period (5 s) Alfvén waves. According to the results of Fig. 4, these waves are reflected in the solar transition region, but once they reach the solar corona, their reflection is negligible. It must be noted that neither the reflection of the $80 \mathrm{~s}$ waves nor the reflection of the $5 \mathrm{~s}$ waves is total; actually, the wave reflection in the atmosphere considered here is always partial (see Sect. 5 for details).

\subsection{Special case of isothermal medium}

A special case of a stratified and isothermal medium with the uniform background magnetic field was considered by Musielak $\&$ Moore (1995). There the density and pressure scale heights are identical, which means that $\Lambda_{d}=$ const (see Eq. (60)). Using Eqs. (56) and (57), we obtain

$\Omega_{\mathrm{cr}, \mathrm{v}}^{2}(y)=-\frac{1}{4} \Omega_{\mathrm{A}}^{2}(y), \quad \Omega_{\mathrm{cr}, \mathrm{b}}^{2}(y)=\frac{3}{4} \Omega_{\mathrm{A}}^{2}(y)$,

which are the same critical frequencies as those obtained by Musielak \& Moore (1995).
Since $\Omega_{\mathrm{cr}, \mathrm{b}}^{2}>\Omega_{\mathrm{cr}, \mathrm{v}}^{2}$, we get the relationship $\Omega_{\mathrm{tp}, \mathrm{b}}^{2}>\Omega_{\mathrm{tp}, \mathrm{v}}^{2}$ for the turning-point frequencies, and obtain the cutoff-frequency

$\Omega_{\text {cut,isoth }}(y)=\left[\Omega_{\mathrm{cr}, \mathrm{b}}^{2}(y)+\frac{1}{4 \tau^{2}(y)}\right]^{1 / 2}$.

Using this cutoff-frequency, we write the condition $\omega>\Omega_{\text {cut,isoth }}$ for the propagation of linear Alfvén waves along the uniform magnetic field oriented along the $y$-axis in a stratified and isothermal medium.

\section{Numerical simulations of the wave equations}

As analytical solutions of Eqs. (16) and (17) are found so far for only a few specific choices of $c_{\mathrm{A}}(y)$ (Didenkulova et al. 2009), we adopt CLAWPACK which is a software package designed to compute numerical solutions to hyperbolic partial differential equations (LeVeque 2002). The simulation region $-0.5 \mathrm{Mm}$ $<y<20 \mathrm{Mm}$ is covered by a uniform grid of $10^{4}$ numerical cells. We verified by convergence studies that this grid well represents the simulation region. We set open (with zero-gradient) boundary conditions at the bottom and top boundaries of the simulation region.

The system of Eqs. (16) and (17) was initially (at $t=0 \mathrm{~s}$ ) perturbed by a Gaussian pulse as

$V_{z}(y, t=0)=\exp \left[-\frac{\left(y-y_{0}\right)^{2}}{w_{y}^{2}}\right], B_{z}(y, t=0)=0$.

We set and hold fixed the initial pulse width $w_{y}=0.3 \mathrm{Mm}$ and consider two cases: (a) waves launched below the transition region at $y_{0}=1 \mathrm{Mm}$; and (b) waves launched above the transition region at $y_{0}=4 \mathrm{Mm}$.

Figure 5 illustrates the wave profiles for the case (a) at $t=10 \mathrm{~s}$ (top panel), $t=60 \mathrm{~s}$ (top middle panel), $t=160 \mathrm{~s}$ (bottom middle panel), and $t=500 \mathrm{~s}$ (bottom panel). Note that $\partial V_{z} / \partial y<0\left(\partial V_{z} / \partial y>0\right)$ at the leading (trailing) part of the initial pulse of Eq. (64). According to Eq. (17) we get $\partial B_{z} / \partial t<0$ $\left(\partial B_{z} / \partial t>0\right)$ there. As a result $B_{z}$ becomes negative (positive) at the wave-front (wave-tail), which is clearly seen in Fig. 5 (top panel). The initial pulse splits into counter-propagating pulses. Downward propagating waves are evident at $t=60 \mathrm{~s}$ (top middle panel). The initial pulse already spread into counter-propagating pulses. The upward propagating pulse partially reflects back into the low chromosphere and during this reflection the wave phase is not reversed. At $t=500 \mathrm{~s}$ (bottom panel) both the reflected and originally propagating downward pulses almost merged into a single quantity. As Eqs. (16) and (17) are coupled any initial signal in $V_{z}$ excites perturbations in $B_{z}$. Indeed, these perturbations are obvious in Fig. 5.

Note that Eq. (19) can be rewritten as

$\frac{\partial^{2} B_{z}}{\partial t^{2}}=\frac{\mathrm{d} c_{\mathrm{A}}^{2}}{\mathrm{~d} y} \frac{\partial B_{z}}{\partial y}+c_{\mathrm{A}}^{2} \frac{\partial^{2} B_{z}}{\partial y^{2}}$.

From Fig. 2 we infer that the gradient of Alfvén speed $\mathrm{d} c_{\mathrm{A}} / \mathrm{d} y$ attains its maximum at the transition region. As a result of that downward propagating perturbations in $B_{z}$ (Fig. 5, dashed line) are much larger than the perturbations in $V_{z}$. Note that in order to compare magnitudes of $V_{z}$ and $B_{z}$ the latter was expressed in $10^{-3}$ Teslas.

Time-signatures which correspond to the wave profiles of Fig. 5 are displayed in Fig. 6. These time-signatures are made by collecting wave signals at the detection point $y=10 \mathrm{Mm}$. 
K. Murawski and Z. E. Musielak: Alfvén waves in the solar atmosphere
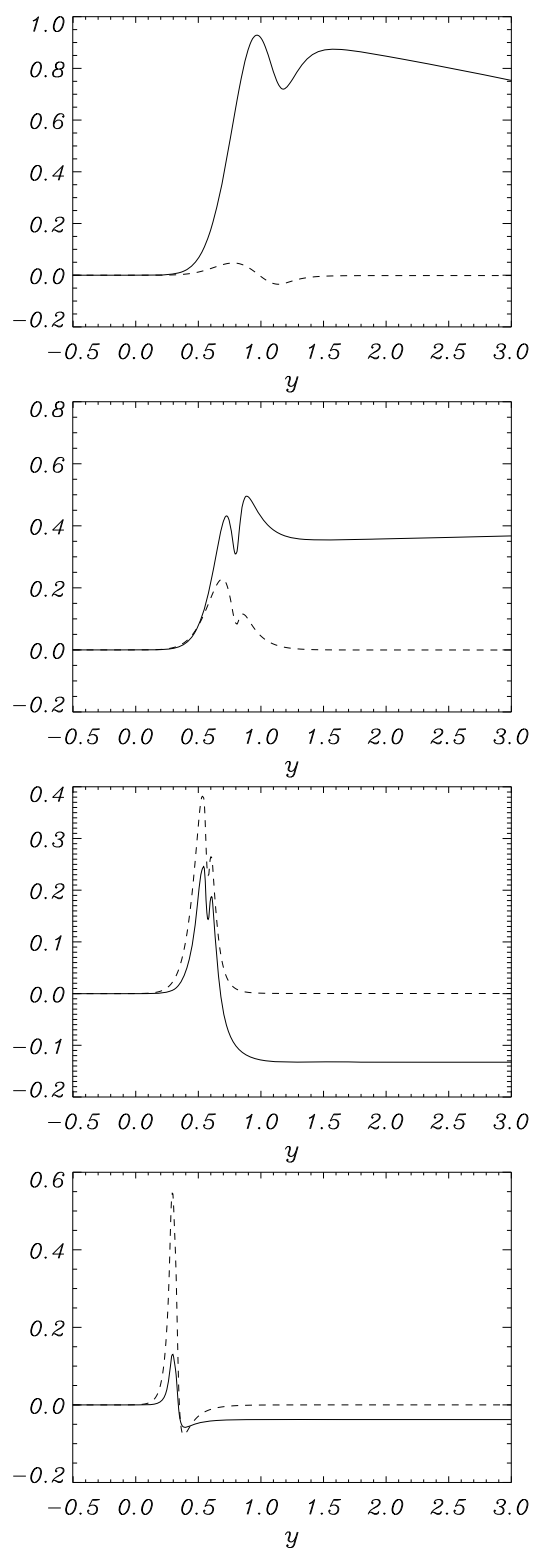

Fig. 5. Wave profiles at (from top to bottom) $t=10 \mathrm{~s}, t=60 \mathrm{~s}, t=$ $160 \mathrm{~s}$ and $t=500 \mathrm{~s}$ for the linear Eqs. (16) and (17) for $y_{0}=1 \mathrm{Mm}$. Solid (dashed) line corresponds to $V_{z}\left(B_{z}\right)$, which is expressed in units of $1 \mathrm{Mm} \mathrm{s}^{-1}\left(10^{-3} \mathrm{~T}\right)$.

These waves passed through the transition region and reached the solar corona. Note that the wave signals grow fast, reaching their maximum at $t \simeq 35 \mathrm{~s}$. Later, the wave signals fall off.

Figure 7 shows a Fourier power spectrum, which reveals that most power is concentrated at the long wave period waves with a maximum located at $P \simeq 500 \mathrm{~s}$, which falls within the range of 3-9 min oscillations detected in the solar corona (Jess et al. 2009; DePontieu et al. 2007; Tomczyk et al. 2007; Bonet et al. 2008; Wedemeyer-Böhm \& van der Voort 2009; Fujimura \& Tsuneta 2009).

Note that the approximate arrival time to the level $y=$ $10 \mathrm{Mm}$ is $t_{\mathrm{app}} \simeq 8 \mathrm{~s}$, which is shorter than $t_{\mathrm{ac}} \simeq 35 \mathrm{~s}$ (Fig. 8) to which the numerical data fits very well.

Now we discuss the case (b). Figure 9 displays wave profiles at three consecutive times. At $t=2 \mathrm{~s}$ (top panel) the initial pulse was already split into counter-propagating pulses. The pulse propagating downwards reaches the transition region at

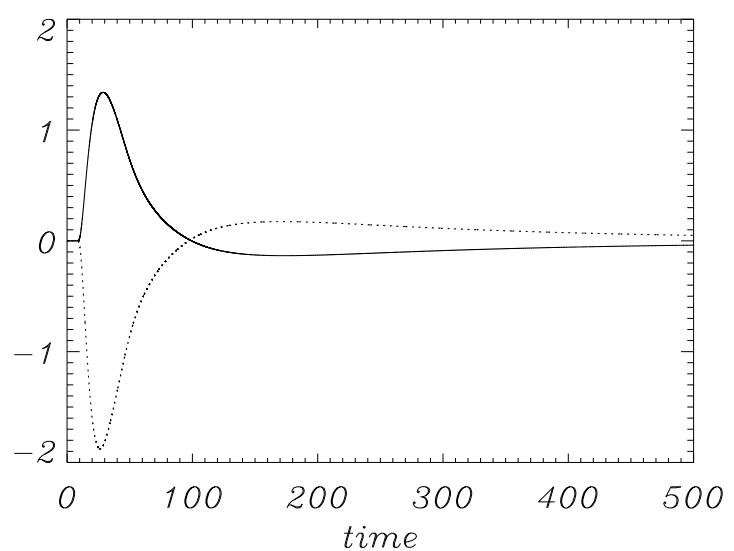

Fig. 6. Time-signatures of $V_{z}$ (solid line) and $B_{z}$ (dashed line) collected at $y=10 \mathrm{Mm}$, resulting from numerical solutions of Eqs. (16) and (17) for $y_{0}=1 \mathrm{Mm}$. Time, $V_{z}$ and $B_{z}$ are expressed in units of $1 \mathrm{~s}, 1 \mathrm{Mm} \mathrm{s}^{-1}$ and $10^{-3} \mathrm{~T}$, respectively.

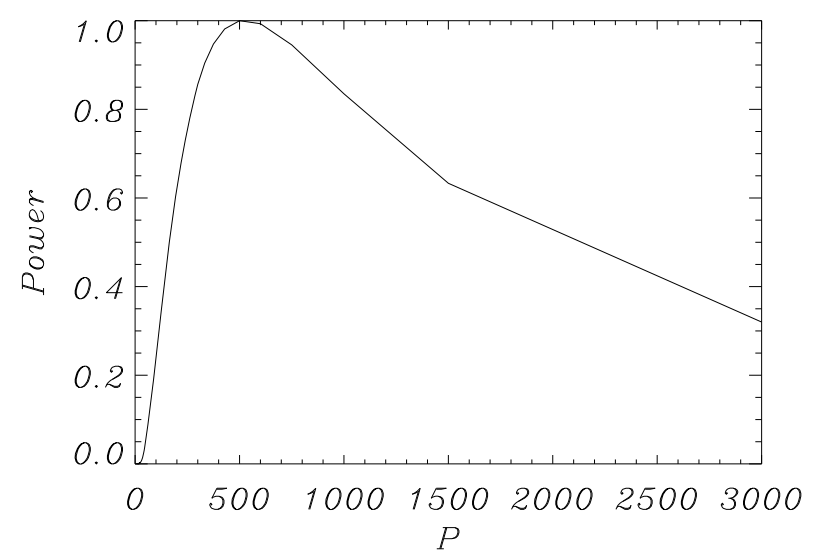

Fig. 7. Normalized Fourier power spectrum. Wave-period $P$ is expressed in $1 \mathrm{~s}$.

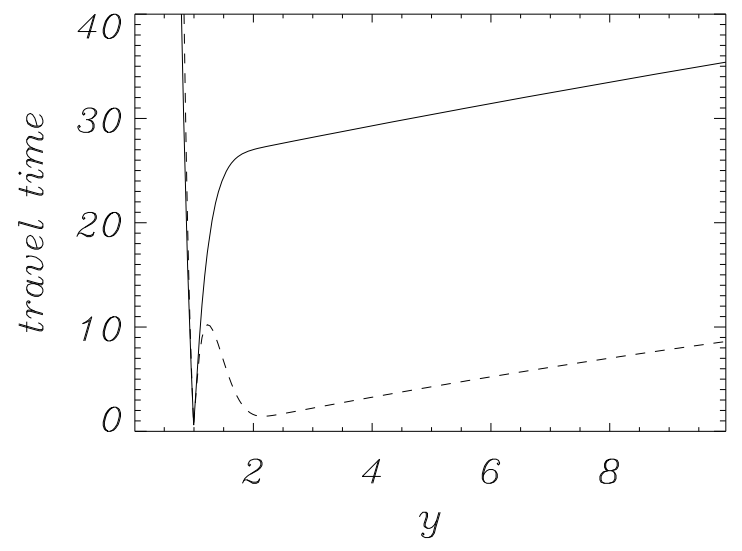

Fig. 8. Actual wave (solid line) and approximate (dashed line) travel times to the detection point $y_{\mathrm{d}}=10 \mathrm{Mm}$ for $y_{0}=1 \mathrm{Mm}$. Time and distance are expressed in units of $1 \mathrm{~s}$ and $1 \mathrm{Mm}$, respectively.

this time. This pulse is partially reflected back into the solar corona and transmitted into the low chromosphere. Note that the reflected pulse reverses its phase, while for (a) the reflected pulse did not experience phase reversal. At $t=5 \mathrm{~s}$ such transmitted and reflected pulses are evident at respectively $y \simeq 1.5 \mathrm{Mm}$ and $y \simeq 4 \mathrm{Mm}$ (middle panel), while the upward propagating signal reaches the height $y \simeq 9 \mathrm{Mm}$. At $t=10 \mathrm{~s}$ the transmitted, 

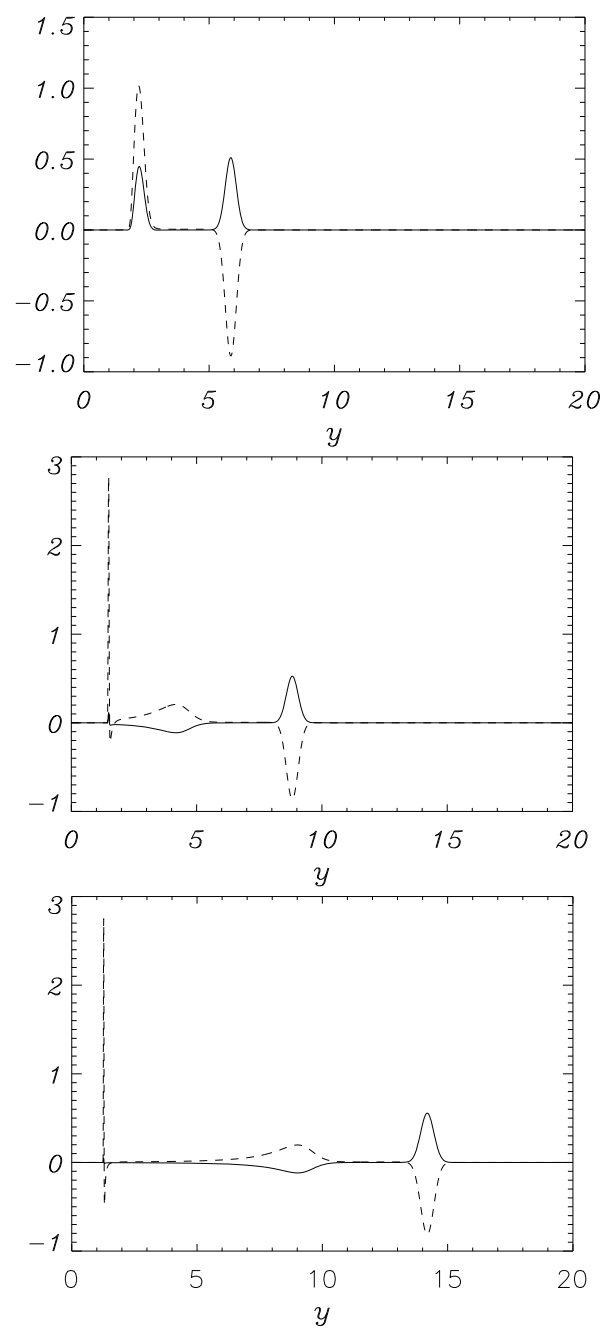

Fig. 9. Wave profiles at $t=2 \mathrm{~s}$ (top panel), $t=5 \mathrm{~s}$ (middle panel) and $t=10 \mathrm{~s}$ (bottom panel) for the linear Eqs. (16) and (17) for $y_{0}=4 \mathrm{Mm}$. Solid (dashed) line corresponds to $V_{z}\left(B_{z}\right)$, which is expressed in units of $1 \mathrm{Mm} \mathrm{s}^{-1}\left(10^{-6} \mathrm{~T}\right)$.

reflected and propagating upwards pulses are at $y \simeq 1.25 \mathrm{Mm}$, $y \simeq 9 \mathrm{Mm}$ and $y \simeq 14 \mathrm{Mm}$, respectively. See bottom panel.

Figure 10 shows time-signatures which are made by collecting wave signals at the detection point $y=1.3 \mathrm{Mm}$. These signatures consist of short period waves with the maximum Fourier power spectrum amplitude located at $P \simeq 5 \mathrm{~s}$ (Fig. 11). These waves have passed into the bottom layer through the transition region. Note that the signal arrives at the detection point at $t \simeq 9 \mathrm{~s}$, which agrees both with the approximate $\left(t_{\mathrm{ap}}\right)$ and accurate $\left(t_{\mathrm{ac}}\right)$ arrival times of Fig. 12.

\section{Discussion of the analytical and numerical results}

We analytically and numerically studied the propagation of linear Alfvén waves in the solar atmosphere. Our model of the solar atmosphere is non-isothermal and its temperature distribution approximates that observed in the solar chromosphere, transition region, and corona. The results of our analytical and numerical studies extend those previously obtained by Musielak \& Moore (1995) and An et al. (1989), respectively, who investigated the Alfvén wave propagation in an isothermal atmosphere.

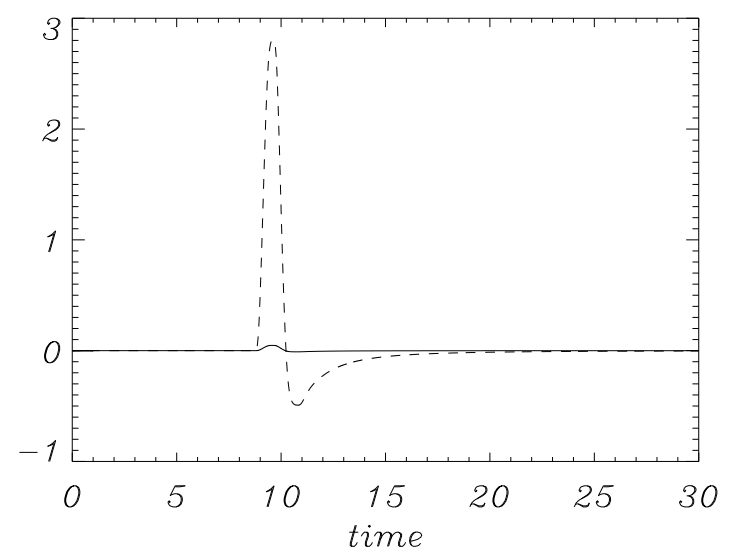

Fig. 10. Time-signatures of $V_{z}$ (solid line) and $B_{z}$ (dashed line) collected at $y=1.3 \mathrm{Mm}$, resulting from numerical solutions of Eqs. (16) and (17) for $y_{0}=4 \mathrm{Mm}$. $V_{z}$ and $B_{z}$ are expressed in units of $1 \mathrm{Mms}^{-1}$ and $10^{-6} \mathrm{~T}$, respectively.

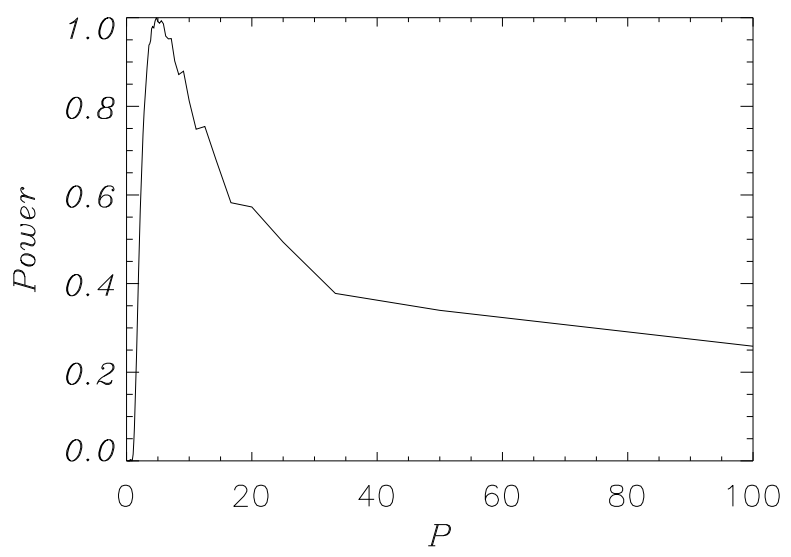

Fig. 11. Normalized Fourier power spectrum vs. wave period $P$ (in 1 s) for the time-signature of Fig. 10.

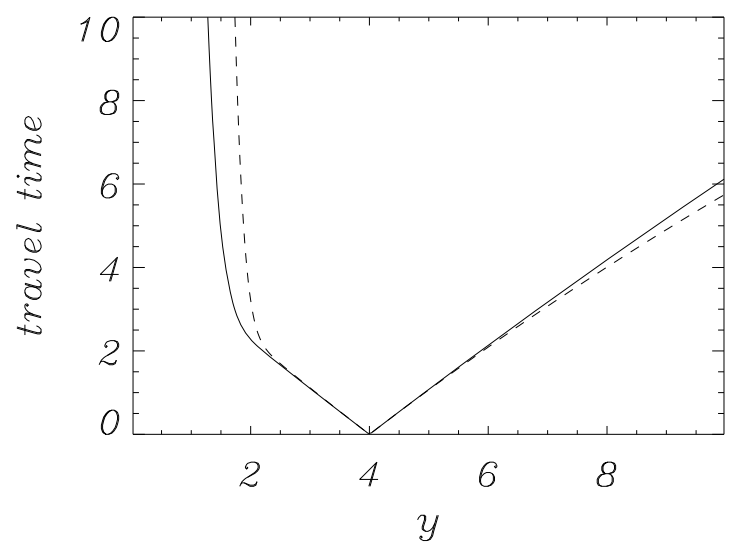

Fig. 12. Actual wave (solid line) and approximate (dashed line) travel times (in units of $1 \mathrm{~s}$ ) to the detection point $y=1.3 \mathrm{Mm}$ for $y_{0}=4 \mathrm{Mm}$. Distance $y$ is expressed in units of $1 \mathrm{Mm}$.

Our main analytical result is the cutoff-frequency (or the cutoff-period $\left.P_{\text {cutoff }}\right)$ obtained with the actual wave travel time. In the model considered here, the cutoff is a local quantity and its variation with height is used to identify regions in the solar atmosphere where strong wave reflection occurs. Using the condition $P_{\mathrm{w}}=P_{\text {cutoff }}$, where $P_{\mathrm{w}}$ is the wave period, the atmospheric height at which Alfvén waves of a given period are reflected is 
established. The condition results from the turning-point property of Euler's equation, and that Alfvén wave reflection is strong at the atmospheric height where the turning-point is located was originally demonstrated by Musielak \& Moore (1995). The analytical solutions obtained by these authors showed that linear Alfvén waves form standing wave patterns in their isothermal model. The reason for the existence of these standing waves is wave reflection and the resulting constructive interference between the propagating and reflected Alfvén waves (see also Musielak et al. 2006).

The formation of the standing wave patterns in the isothermal solar atmosphere was numerically investigated by An et al. (1989), who were able to reproduce full analytical solutions by their numerical simulations and identify a height in their atmospheric model at which Alfvén wave reflection is dominant. Their main result is that wave reflection occurs continuously in the isothermal atmosphere and that it reaches maximum at the height where the wave period becomes comparable to a local cutoff-period. Their results also clearly demonstrate that wave reflection is always partial, even at its maximum. We extended the previous work by considering a more realistic nonisothermal solar atmosphere and investigating impulsively generated linear Alfvén waves.

Our main numerical results are presented and discussed in Sect. 4, so here we only comment on their relationship to our analytical results. The analytical predictions of the atmospheric height at which strong reflection occurs for waves of a given period and the numerical results agree well. Moreover, the numerical results also clearly show that wave reflection is only partial, even at the height where it reaches its maximum. This confirms the previous analytical (Musielak \& Moore 1995) and numerical (An et al. 1989) results. Unfortunately, for the non-isothermal solar atmosphere model considered here, we were unable to obtain full analytical solutions to the Alfvén wave equations. As a result, we were unable to make directly compare between analytical and numerical solutions obtained for very long simulation times. We hope that this comparison would further validate our results.

\section{Conclusions}

We studied small-amplitude Alfvén waves propagating in the stratified solar atmosphere. We particularly considered impulsively triggered waves in the low and top layers of the solar atmosphere. Guided by this we derived the transformed wave equations for the transversal velocity and magnetic field components which correspond to linear Alfvén waves. From these equations we obtained the cutoff-wave period $P_{\text {cutoff }}$, which varies with height in the solar atmosphere. We used the cutoff to determine the regions in the solar atmosphere where the reflection of Alfvén waves becomes dominant.

The analytical findings were subsequently verified by numerical solutions of the wave equations. The numerical results for impulsively generated waves revealed that the Alfvén waves exhibit characteristic spatial and temporal signatures, which agree with the analytical predictions. Specifically, the analytically predicted height where Alfvén wave reflection dominates in the wave behavior, agrees with the numerical results. Moreover, the numerical simulations clearly show that wave reflection is always partial, even at the height where it reaches its maximum.

The results presented here are hopefully enable a deeper understanding of the complex relation between Alfvén waves and transient events in the solar chromosphere and corona. This work paves the way for future studies and it serves as a reference to the more advanced numerical solutions of the MHD equations, which will be reported elsewhere. In particular, a more realistic model of the solar atmosphere has to be taken into consideration. In this model a more appropriate temperature profile than the simple model of Eq. (11) as well as non-ideal plasma effects and neutral plasma components in the photosphere have to be implemented.

Acknowledgements. The authors are grateful to the referee for his/her stimulating comment, which resulted in an improvement of the presentation of the results. K.M. appreciates a fruitful discussion with Prof. Valery Nakariakov. The work of K.M. was supported by the Polish Ministry of Science (the grant for years 2007-2010). Z.E.M. acknowledges the support of this work by NSF under grant ATM-0538278, the Alexander von Humboldt Foundation, and the University of Texas at Arlington through its Faculty Development Leave Program.

\section{References}

An, C.-H., Musielak, Z. E., Moore, R. L., \& Suess, S. T. 1989, ApJ, 345, 597 Bonet, J. A., Marquez, I., Sanchez Almeida, J., Cabello, I., \& Domingo, V. 2008, ApJ, 687, 431

De Pontieu, B., McIntosh, S.W., Carlsson, M., et al. 2007, Science, 318, 1574

Didenkulova, I., Pelinovsky, E., \& Soomere1, T. 2009, JGR, 114, C07006

Fujimura, D., \& Tsuneta, S., 2009, ApJ, 702, 1443

Hollweg, J. V. 1991, in Mechanisms of Chromospheric and Coronal Heating, Proceedings of the International Conference, Heidelberg, 5-8 June 1990, XV, 649, 260 figs, ed. P. Ulmschneider, E. R. Priest, \& R. Rosner (Heidelberg, New York: Springer-Verlag), 423

Hollweg, J. V., \& Isenberg, P. A. 2007, JGR, 112, A08102

Jess, D. B., Mathioudakis, M., Erdélyi, R., et al. 2009, Science, 323, 1582

Kahn, P. B. 1990, Mathematical Methods for Scientists and Engineers (New York: John Wiley)

LeVeque, R. J. 2002, Finite Volume Methods for Hyperbolic Problems (Cambridge University Press)

Matsumoto, T., \& Shibata, K. 2010, ApJ, 710, 1857

Murphy, G. M. 1960, Ordinary Differential Equations and Their Solutions, D. Van Nostrand (New York: Company)

Musielak, Z. E., \& Moore, R. J. 1995, ApJ, 452, 434

Musielak, Z. E., Rosner, R., \& Ulmschneider, P. 1987, in Cool Stars, Stellar Systems and the Sun, ed. J. L. Linsky, \& R. E. Stencel, Lect. Notes Phys., 291, 66

Musielak, Z. E., Fontenla, J. M., \& Moore, R. L. 1992, Phys. Fluids, 4, 13

Musielak, Z. E., Musielak, D. E., \& Mobashi, H. 2006, Phys. Rev. E, 73, 036612-1

Parker, E. N. 1991, ApJ, 376, 355

Rae, I. C., \& Roberts, B. 1982, ApJ, 256, 761

Routh, S., Musielak, Z. E., \& Hammer, R. 2010, ApJ, 709, 1297

Suzuki, T. K., \& Inutsuka, S.-I. 2005, ApJ, 632, L49

Tomczyk, S., McIntosh, S. W., Keil, S. L., et al. 2007, Science, 317, 1192

Van Doorsselaere, T., Brady, C. S., Verwichte, E., \& Nakariakov, V. M. 2008a, A\&A, 491, L9

Van Doorsselaere, T., Nakariakov, V. M., \& Verwichte, E. 2008b, ApJ, 676, L73

Velli, M. 1993, A\&A, 270, 304

Verdini, A., \& Velli, M. 2007, ApJ, 662, 669

Verdini, A., Velli, M., \& Buchlin, E. 2009, ApJ, 700, L39

Zaqarashvili, T. V., \& Murawski, K. 2007, A\&A, 470, 353

Wedemeyer-Böhm, S., \& van der Voort, L. R. 2009, A\&A, 507, L9 